\title{
The Mechanism Study of Alternating Arc (AC) Magnetic Levitation Induction Motor
}

\author{
Zeng Li \&Chen Qiuyue \&Chen Fang \&Zhang Fan \\ College of Mechanical Engineering, Yangzhou University, Yangzhou 225127, China \\ E-mail: lizengcf@163.com,13665203656@163.com
}

\begin{abstract}
Magnetic levitation (no bearings) motor by using magnetic force to make rotor suspend and drive realize its high or ultra high speed rotating. The stator's structure of traditional no bearing magnetic levitation motor is double winding which is polar logarithmic difference 1 of 2 sets of winding (torque winding and suspension winding) and embedded in the stator. Using two inverter respectively for the two sets of winding to go into the same frequency current in order to realize the suspension of the rotor and motor's driven, small carrying capacity of motor's structure, controlling complex system. This paper based on the traditional motor technology puts forward a kind of arc principle and respectively decorates two arc motors in horizontal and vertical direction symmetric to rotor according to the electromagnetic bearing suspension technology that is constituted the arc magnetic levitation induction motor. Establishing air-gap transformation regular between rotor and stator (air-gap length) motor is under the effect of interference. Based on the electromagnetic theory establishing distribution regular of the air-gap magnetic induction intensity. Virtual displacement principle is used to establish electromagnetism mathematical model and motor electromagnetism levitation. By the finite element analysis carrying on simulation research to the magnetic induction intensity, electric magnetic levitation force and distribution features of electromagnetic torque and so on.
\end{abstract}

KEYWORD: Alternating arc motor; Magnetic induction intensity; Levitation force features; Electromagnetic torque feature

\section{INTRODUCTION}

Magnetic levitation motor devotes the winding produced radial force in the electromagnetic bearing to overly on the motor stator's winding that is formed two different logarithmic rotating magnetic field in air-gap so as to produce torque and levitation force at the same time and realize the motor's driven and rotor's self-suspension[1]. Stator of magnetic levitation motor has double winding and single winding .At present, most of the magnetic levitation motor stator is double winding which is polar logarithmic difference 1 of 2 sets of winding (torque winding and suspension winding) and embedded in the stator. Using two inverter respectively for the two sets of winding to go into the same frequency current in order to realize the suspension of the rotor and motor's driven[1-2].But bearing capacity of this kind of motor's structure is small and control system of that is complex. For this, put forward a new kind of alternating arc magnetic induction motor based on supporting and driving of the stator of 4 arc motor. It is same to the winding structure of traditional motor, used to modify the balance of magnetic field produced by Arc motor stator's winding to produce levitation force realizing rotor's self-suspension. Compared to common double winding magnetic levitation motor, its structure is simple and the loss of motor's capacity factor is small[3]. This paper related to alternating arc magnetic levitation motor has studied rotating and suspension mechanism, establish mathematical model of system. By the finite element analysis and experiment method studying motor's suspension and rotating features.

\section{THE MECHANISM OF ALTERNATING ARC MAGNETIC INDUCTION MOTOR}

Ac arc magnetic induction motor's rotor is together supported to suspend and drive to rotate by 4 three mutually alternating arc motor. Arc motor based on traditional motor technology, which is rotated coil winding in wound arc stator and charged with electricity so that make rotor rotating. Analogy to traditional motor, divided to 3 phase, 2 phase or 
single alternating motor (synchronism or asynchronous), straight motor, Dc motor, Stepper (reluctance) motor. Phase ac arc motor stator's winding creates a rotating magnetic field, to cut closed loop winding of rotor and produce induction current and potential, to achieve the rotor rotation.

Ac arc magnetic levitation induction motor is consisted by the stator, rotor, suspending and rotating detection system. Motor based on electromagnetic bearing suspension technology, on the traditional motor stator core, is winded two ac arc 3 phase motor winding symmetrical to rotor in horizontal and vertical direction. Under the comprehensive test control system, differentially modify current increment size of both sides arc motor in the same direction so as to change magnetic force size of rotor which is did by 2 arc motor and make the rotor always working in the direction of center equilibrium position of 2 arc motors. As shown in Figure 1 each set of three phase winding connection of type $\mathrm{Y}$ and current status in a certain time. 4 period of arc stator's windings respectively are sym-metrically arranged 2 circular arc in the $\mathrm{x}$ axis and the $\mathrm{y}$ axis. 3 phase stator winding of 4 period of arc motor which is paralleled to access three phase alternating current i1, i2, i3, respectively closed-loop controlling to current amplitude size of each set of arc motor stator winding, realizing the regulation of rotor's electromagnetic force, produce controllable electromagnetic levitation force. Its working principle of the rotor suspension is same to traditional electromagnetic bearing mechanism[4] , under the effect of interference, the rotor is offset $\Delta \mathrm{x}$ along the $\mathrm{x}$ (right) direction in order to restore the rotor to center position (balance) of the stator. Under the effect of closed loop controlling system, the current of the arc motor stator winding on the left side of rotor increases $\Delta i_{k x} \quad(\mathrm{k}=1,2,3)$ into $i_{k x}^{\prime}=i_{k}+\Delta i_{k x} \quad(k=1,2,3)$, the rotor on the right side of the arc current of motor stator winding decreases $(\mathrm{k}=1,2,3)$, the current of the arc motor stator winding on the right side of rotor decreases $\Delta i_{k x} \quad(\mathrm{k}=$ $1,2,3)$ into $i_{k x}=i_{k}-\Delta i_{k x} \quad(k=1,2,3)$. Figure 2 is 4 synthetic rotating magnetic flux produced by 4 period of arc motor winding.

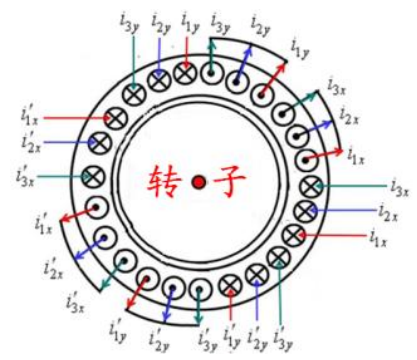

Figure 1. Principle diagram of electrifying magnetic flux.

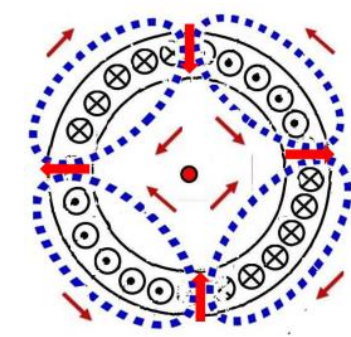

Figure 2. Rotating magnetic flux of ac arc magnetic motor levitation.

\section{SUSPENSION AND ROTATING MODEL OF AC ARC MAGNETIC LEVITATION INDUCTION MOTOR}

\subsection{Change regulation of air-gap length between stator and rotor}

If rotor is column, Figure 3 is schematic diagram when rotor happens to displacement, the air-gap length $\delta(\theta)$ between outer surface of rotor and inner surface of stator the stator can be expressed eccentric distance in the $\mathrm{x}$ and $\mathrm{y}$ directions. Supposing $\mathrm{a}$ unilateral average air-gap length $g_{0}=\mathrm{R}-\mathrm{r}$, rotor offsets $\rho$ along some $\left(\theta_{0}\right)$ direction. The Figure 3 shows $\rho \cos \left(\theta-\theta_{0}\right)+r+\delta(\theta)=R$, so the air gap length of any position in space is

$$
\delta(\theta)=g_{0}-(x \cos \theta+x \sin \theta)
$$

In the formula, $\theta$ is the space vector Angle, $\mathrm{x}$ and $\mathrm{y}$ are the displacement when rotor along the $\mathrm{x}, \mathrm{y}$ direction.

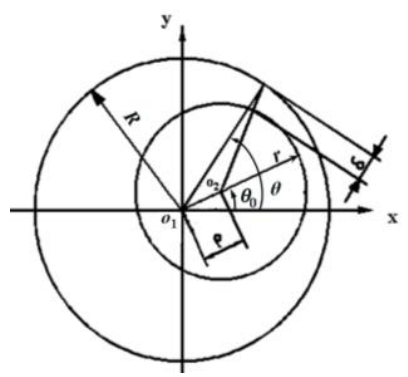

Figure 3. Eccentricity schematic magnetic diagram of rotor

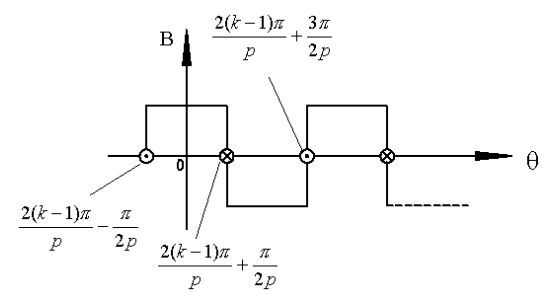

Figure 4. Distribution curve of induction intensity

\subsection{Motor air-gap magnetic induction intensity distribution}

According to the theory of magnetic field , under the action of alternating current $\mathrm{i}(\mathrm{t})$, single-phase 
winding of $\mathrm{p}$ pairs of magnetic pole, magnetic induction intensity of the rotating magnetic field in the circumference of air-gap effective length $g$ is rectangular distribution[5], Figure 4 can gain the distribution of air-gap magnetic induction intensity

$$
B(\theta, t)= \begin{cases}\frac{N i(t) \mu_{0}}{2 g} & \frac{2(k-1) \pi}{p}-\frac{\pi}{2 p} \leq \theta<\frac{2(k-1) \pi}{p}+\frac{\pi}{2 p} \\ -\frac{N i(t) \mu_{0}}{2 g} & \frac{2(k-1) \pi}{p}+\frac{\pi}{2 p} \leq \theta \leq \frac{2(k-1) \pi}{p}+\frac{3 \pi}{2 p}\end{cases}
$$

In the formula, $\mathrm{k}$ is natural Number; $\mu 0$ is the permeability of magnetic field air, $\mu_{0}=4 \pi \times 10^{-7} \mathrm{H} / \mathrm{m}$; $\mathrm{N}$ is number of stator winding; $\mathrm{g}$ is the air-gap length between the stator and rotor.

$$
\begin{aligned}
B(\theta, t)= & \left(\frac{N I \mu_{0}}{2 g} \cdot \frac{4}{\pi}\right)\left(\cos p \theta-\frac{1}{3} \cos 3 p \theta\right. \\
& \left.+\frac{1}{5} \cos 5 p \theta+\cdots\right) \cos \omega t
\end{aligned}
$$

In the formula, I is current amplitude, $\omega_{\text {is current }}$ frequency. Three phase winding of 4 period of circular arc stator is composed of three phase windings, and symmetrical distribution in the motor stator, each of single phase winding produces induction intensity of pulse vibration. The axis of three phase winding of all the motor stator winding composed, is successively separated electrical angle $120^{\circ}$ in space, by the formula (4) after ignoring higher harmonic, the distribution of magnetic induction intensity of the fundamental wave in space is successively separated electrical angle $120^{\circ}$, by type (4) gaining

$$
\left\{\begin{array}{l}
B_{A}(\theta, t)=B_{m} \cos p \theta \cos \omega t \\
B_{B}(\theta, t)=B_{m} \cos \left(p \theta-120^{\circ}\right) \cos \left(\omega t-120^{\circ}\right) \\
B_{C}(\theta, t)=B_{m} \cos \left(p \theta-240^{\circ}\right) \cos \left(\omega t-240^{\circ}\right)
\end{array}\right.
$$

Among them, the magnetic induction intensity value is

$$
B_{m}=\frac{2 \mu_{0} N I}{\pi g}
$$

Its size is depends on the input current of winding and air-gap length. By type (5) calculating magnetic induction intensity of three-phase fundamental wave synthesis produced by all the three-phase winding magnetic potential in the stator's and rotor's magnetic field air-gap is

$$
B_{s}(\theta, t)=B_{m} \cos (\omega t-p \theta)
$$

In this formula, $\theta$ is tangential angle of reeling stator axis; $\mathrm{p}$ is magnetic flied pole logarithmic produced by winding.

Magnetic flux of inductive magnetic levitation motor stator winding rotates and cuts the armature winding of rotor in space, producing inductive electromotive force and the current in the rotor. By alternating current in the rotor can produce spinning magnetic flux in the air gap all the same, its magnetic inductive intensity is

$$
B_{r}(\theta, t)=\beta B_{m} \cos (\omega t-p \theta-\alpha)
$$

In this formula, $\beta$ is inductance coefficient of stator and rotor winding; $\alpha$ is phase difference between magnetic field and winding rotating magnetic field of rotor and stator (including rotor angular displacement). $\beta$ 、 $\alpha$ each of them is decided by of rotor and stator structure and slip ratio of inducting magnetic levitation motor.

By the winding of stator and rotor produced total magnetic inductive intensity on the circumference of the air gap of are

$$
B(\theta, t)=B_{m}(\cos (\omega t-p \theta)+\beta \cos (\omega t-p \theta-\alpha))
$$

Above the type, the distributive regulation of airgap magnetic inductive intensity of inducting magnetic levitation motor is related to magnetic pole logarithm, tangential angle position around the axis of the stator, as well as the phase difference between the rotor and the stator rotating magnetic field produced by the winding of stator.

\section{ANALYSIS OF FINITE ELEMENT SIMULATION OF AC ARC MAGNETIC LEVITATION INDUCTION MOTOR}

Structure parameters of ac arc magnetic levitation induction motor have shown in table 1. By the parameters of table 1 building geometry model of magnetic levitation motor stator with rotor and mesh, Figure 5 is the air-gap enlargement chart. After the finite element calculation, can get the initial distribution of magnetic force lines, as shown in Figure 6.

Figure7 is distributive chart of magnetic flux density after running a spin cycle. Magnetic inductive intensity in the middle of the stator and rotor magnetic circuit with the air-gap distribute more uniform, magnetic flux density in the stator tooth is bigger, near the edge of rotor and outer of stator appear to magnetic leakage in a certain extent, but not obvious.

Figure8 is distributive chart of magnetic pole airgap magnetic flux density produced by a set of circular motor winding. Due to the actual tooth slot, magnetic leakage and other factors for example edge effect, so the magnetic flux density is fluctuating, the average effect is fundamental with the cosine regulation distribution, equal to the theoretical value. 


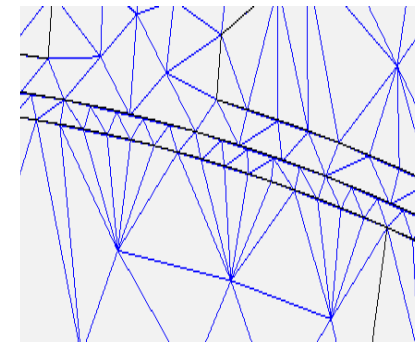

Figure 5

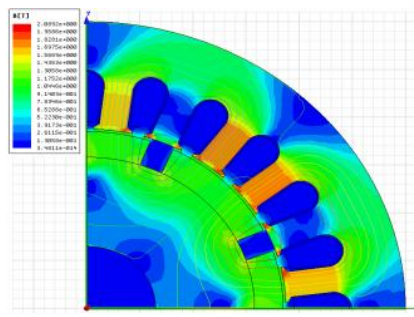

Figure 7

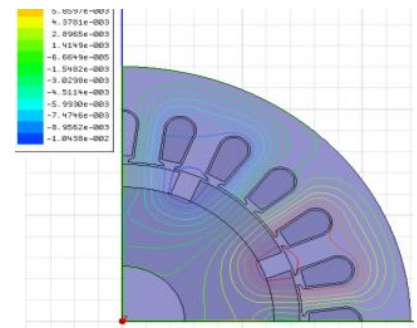

Figure 6

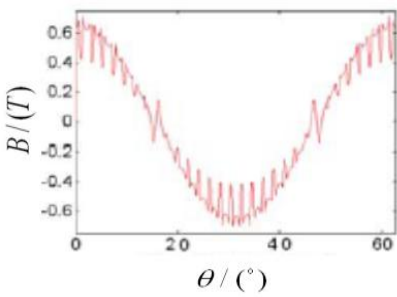

Figure 8
Table 1.Model parameters of ac arc magnetic levitation induction motor

\begin{tabular}{|l|l|}
\hline Parameter name & Parameter values \\
\hline Inner diameter of stator & $64.5 \mathrm{~mm}$ \\
\hline Outer diameter of stator & $75 \mathrm{~mm}$ \\
\hline Tooth width & $14 \mathrm{~mm}$ \\
\hline Air-gap length & $0.25 \mathrm{~mm}$ \\
\hline Axial length & $20 \mathrm{~mm}$ \\
\hline $\begin{array}{l}\text { Numbers of winding (A set of three } \\
\text { phase winding) }\end{array}$ & 130 \\
\hline
\end{tabular}

\section{THE CONCLUSION}

1) This paper based on technology of the traditional motor is proposed principle of arc motor, according to the suspension mechanism of electromagnetic bearing, ac three phase-4 circular arc magnetic levitation motor is consisted by arc motor, analyzed the basic composition and working principle of the motor;
2) Setting up coordinates changing regulation and the and air-gap magnetic field distribution model of air-gap length of ac arc magnetic levitation induction motor;

3) Based on air-gap magnetic field distribution of ac arc magnetic levitation induction motor, the virtual displacement principle is used to establish the electromagnetic levitation force and electromagnetic torque model of motor;

4)By the finite element simulation analysis verifying air-gap magnetic induction intensity, electric-magnetic levitation force and electromagnetic torque distribution characteristics of ac arc magnetic levitation induction motor.

\section{ACKNOWLEDGEMENTS}

China Natural Science Foundation for this project. (NO. 51375427); Jiang su Natural Science Foundation for this project (NO.BK20131232); Yangzhou city and Yangzhou university cooperation projects (NO.YZ2011152).

\section{REFERENCES}

[1] CHIBA A, DEIDO T, FUKAO T, et al. An analysis of bearing-less AC motors. IEEE Transactions on Energy Conversion, 1994, 9(1):61-68.

[2] NIAN HENG, HE Y-IKANG. Analytical modeling and feedback control of the magnetic levitation force for an induction-type bearing-less motor. Proceedings of the CSEE, 2003, 23(11):139-144.

[3] KHOO W K S. Bridge configured winding for poly phase self-bearing machines. IEEE Transactions on Magnetics, 200541 (4):1289-1295.

[4] ZengLi, Zhu Hangqiu. The Study on Permanent Magnet Biased HMB, CHINA MECHANICAL ENGINEERING, 1999.4(4): 387-389.

[5] Chen Pizhang, Yan Lietong. Motor electromagnetic field theory and calculation, Science Press, 1986.3. 